\title{
Ultrastructural and immunocytochemical analysis of the XY body in rat and Guinea pig
}

\author{
0.M. Echeverría, ${ }^{*}$ R. Benavente, ${ }^{\circ}$ R. Ortiz, ${ }^{*}$ G.H. Vázquez-Nin* \\ *Laboratory of Electron Microscopy, Department of Cell Biology, Faculty of Sciences, National University \\ of Mexico, Mexico D.F. Mexico; ' Department of Cell and Developmental Biology, Biocenter of the \\ University of Wurzburg, Wurzburg, Germany
}

(C)2003, European Journal of Histochemistry

The formation of the XY body involves the compaction of the extended chromatin to form a mesh of fibrogranular structures. During this process the ribonucleoprotein particles (RNP), which were associated with the chromatin filaments progressively disappear. High resolution immunolocalization indicates that the mature XY body does not contain RNA polymerase II, hnRNPs, or snURNPs. Occasionally chromatin fibrils extend outside of the $X Y$ body. These fibrils are frequently associated with nascent RNP fibrils and granules indicating that not all the DNA of the sex chromosomes is transcriptionally inactive. However, transcription is located outside the sex body. The recombination protein Dmc1 is present in nodules associated with the unpaired chromosomal axes of the sex chromosomes located in the XY body. Cytochemical staining methods and in situ hybridization at electron microscopic level show that RNA is present in the unpaired chromosomal axes suggesting that the presence of RNA in the chromosomal axes and in forming synaptonemal complexes is related with the process of final pairing. The sex body and the nucleoli associated with it do not interweave and do not exchange RNA or DNA-containing filaments. These observations indicate that the spatial relation between these structures is just a close proximity, which is, however, very frequent.

Key words: sex vesicle, RNA, transcription, synaptonemal complex.

Correspondence. Dr. Olga M. Echeverría, Apartado Postal 70438, México D.F., México. Phone: international +52.55 . 56224881. Fax: international +52.55.56224828.

E-mail: omem@hp.fciencias.unam.mx

Paper accepted on June 26, 2002

European Journal of Histochemistry 2003; vol. 47 issue 1 [Jan-Mar]:45-54
During the pachytene stage of the male meiotic prophase in mammals, the sex chromosomes become partially condensed to form the $X Y$ body (Solari, 1974). In oogenesis the chromatin of the sex chromosomes behaves as that of autosomes (Solari, 1974). The XY body possesses several peculiarities: a) it is formed by a mass of condensed chromatin and two chromosomal axes of different length; $b$ ) in contrast to the autosomes, the $X Y$ body is transcriptionally inactive (Kierszenbaum and Tres, 1974; Solari, 1989); c) recombination is restricted to a small subtelomeric region of the chromosomes; d) the mechanism by which transcription becomes inactive differs from the one involved in X chromosome inactivation in the interphase of somatic cells (Armstrong et al., 1997; Marahrens et al., 1997; Bauer et al., 1998).

Several studies combining biochemical and immunocytochemical methods at light microscopic level have characterized $X Y$ body associated proteins. Some of these proteins are probably related with transcription inhibition, chromatin condensation, and other distinctive features of this body (Smith and Benavente, 1992; Smith and Benavente, 1995; Kralewski and Benavente, 1997; Turner et al., 2000).

Recombination proteins as Rad51/Dmcl induce the formation of double strand breaks and their repair, which are the most accepted mechanisms of nonsister DNA-DNA interactions. They also promote in vitro homologous pairing and strand exchange reactions in human (Benson et al., 1994; Baumann et al., 1996). Recognition of homologous sequences is frequently attributed to the formation of duplexes of complementary nonsister DNA molecules (Smithies and Powers, 1986; Loidl, 1990; Kleckner et al., 1991; Kleckner and Weiner, 1993; Moens 1994; Weiner and Kleckner, 1994; Kleckner, 1996; McKee, 1996). Also, the alignment of chromosomes was proposed to result from homology search initiated by the formation of double strand 
breaks (reviewed by Moens, 1994). However, RNA may also be related with homology recognition and pairing. Transcription and chromosome alignment are related phenomena. There is evidence that chromosomes only pair when they are transcriptionally active (Cook, 1997). Effective pairing systems are primarily associated with coding sequences, which excludes constitutive and facultative heterochromatin and non-heterochromatic repetitive DNA sequences from initial pairing (Sybenga, 1999). The study of RNA localization using classical contrast techniques and in situ hybridization, as well as the immunolocalization of RNA polymerase II and proteins associated with $m R N A$, may contribute to the knowledge of the relation of homology search, pairing and transcription in the $X Y$ body.

Electron microscopic studies have scarcely contributed to the knowledge of the cytophysiological role of the $X Y$ body after its three-dimensional reconstruction by Solari and Tres (Solari and Tres, 1970), that demonstrated the presence of a short synaptonemal complex (SC) in continuity with two diverging chromosomal axes of different length. This observation in situ demonstrated the partial pairing between human $X$ and $Y$ chromosomes. The fine localization of DNA in the $X Y$ body using classical and immunocytochemical methods may establish whether the degree of compactness of the chromatin of the allocyclic sex chromosomes is similar to interphase compact chromatin or to some intermediary stages present during large changes of transcriptional activity (Vázquez-Nin et al., 1978).

The aim of the present work was to determine the presence and distribution of DNA, RNA, recombination proteins, and proteins related to transcription and its products in the $X Y$ body, using high resolution cytochemical and immunocytochemical methods.

\section{Materials and Methods}

Testes of young adult rat and guinea pigs were used in all experiments. The standard procedure for preparing the samples involves fixation in $2.5 \%$ glutaraldehyde in $0.2 \mathrm{M}$ phosphate buffer $\mathrm{pH} 7.3$ for $1 \mathrm{~h}$ at room temperature, rinsing in the same buffer, dehydration with ethanol, and embedding in an epoxy resin (Glycide ether 100, Merck, Darmstadt, Germany). One micrometer thick sections were stained with toluidine blue. The cellular associations seen in seminiferous tubules were compared with the classical descriptions of the sper- matogenic cycle of the guinea pig (Clermont, 1960) and of the rat (Leblond and Clermont, 1952).

\section{Three-dimensional reconstructions}

Samples of testis were fixed in $2.5 \%$ glutaraldehyde and embedded in Epon. Once a stage of meiotic prophase was selected in light microscope, serial ultrathin sections were carried out. Ribbons of sections were picked up with uncoated slot grids, which were mounted on Formvar film as described by Wettstein and Graver (1973). The third dimension was reconstructed by superposing transparent photographic images or transparent traces of successive sections. In order to stain RNP structures, the uranyl acetate-EDTA-lead citrate procedure (Bernhard, 1969) and terbium method (Biggiogera and Fakan, 1998), were used. DNA was detected using the osmium-ammine procedure (Cogliati and Gautier, 1973) modified by Vázquez-Nin and coworkers (1995).

\section{Immunolocalization}

Samples of seminiferous tubules were fixed in 2$4 \%$ paraformaldehyde in $0.15 \mathrm{M}$ phosphate buffer $\mathrm{pH} 7.3$ and embedded in Lowicryl K4M. Polymerization was carried out using UV at $-20^{\circ} \mathrm{C}$. The ultrathin sections were mounted in Formvar-coated nickel grids, and processed for postembedding immunolocalization as described previously (Biggiogera et al., 1989; Vázquez-Nin et al., 1990). The following antibodies were used for immunolocalization:

- anti-RNA polymerase II rabbit polyclonal antibody (Kim \& Dahmus 1986);

- anti-DNA mAb (Progen, Heildelberg, Germany);

- anti-hnRNP core protein (C8V, Jones et al. 1980);

- anti-Trimethyl-guanosin cap (R1131, Oncogene, New York, USA);

- $2 \mathrm{H} 12$, monoclonal anti-human Dmcl Ab.

\section{Ultrastructural in situ hybridization}

Specimens were fixed in $2 \%$ paraformaldehyde or in $4 \%$ paraformaldehyde with $0.2 \%$ glutaraldehyde added and embedded in Lowicryl K4M (Polysciences, USA).

Genomic DNA from guinea pig liver and spleen was extracted according with López-Velázquez et al. (1998). Final concentration of the DNA was 5 $\mathrm{mg} / \mathrm{mL}$.

The DNA was labeled with digoxigenin-11-dUTP 
by nick translation according with the manufacturer's protocol (Boehringer Mannheim, Germany). The size of the probe was estimated by electrophoresis in agarose gels.

The denaturation solution contained: $2 \mathrm{~mL}$ of the labeled probe $(5 \mathrm{mg} / \mathrm{mL})$, with $4 \mathrm{mg}$ of tRNA (Boehringer Mannheim, Germany), and $10 \mathrm{~mL}$ of formamide (Gibco-BRL, USA) added. Denaturation was carried out at $90^{\circ} \mathrm{C}$ for $4 \mathrm{~min}$, followed by rapid cooling in ice.

The in situ hybridization was performed on sections mounted on Formvar-coated gold grids without denaturation of the DNA of the sample. The probe was used at $0.4 \mathrm{mg} / \mathrm{mL}$ final concentration in a hybridization solution containing $42 \%$ formamide, 4 mg tRNA, 0.8\% BSA, 8.3\% Dextran sulfate, and 1.7XSSC (Sigma, St. Louis Missouri, USA). The hybridization reaction was performed at $42^{\circ} \mathrm{C}$ overnight in a moist chamber. The hybridized probe was detected by incubation of the grids with a rabbit anti-digoxigenin (Sigma, St. Louis, Missouri, USA). The anti-digoxigenin was localized with a goat anti-rabbit IgG (Dako, Denmark). This IgG was recognized with protein A coupled with 10 $\mathrm{nm}$ gold grains (Auroprobe, USA). In order to amplify the labeling, two further antibodies were employed: a GAR IgG $(H+L)$ (Auroprobe, USA), and RAG IgG (Sigma, St. Louis Missouri, USA), both coupled with $10 \mathrm{~nm}$ gold grains.

To confirm the RNA nature of the hybridization signal, some sections were pre-treated with 2 $\mathrm{mg} / \mathrm{mL}$ ribonuclease (type IA, Sigma) in $1 \mathrm{mM}$ triethanolamine-acetic acid buffer, $\mathrm{pH} 7,3$ for 8 hours at $37^{\circ} \mathrm{C}$.

All preparations were contrasted with $2.5 \%$ uranyl acetate for $5 \mathrm{~min}$ and lead citrate for $1 \mathrm{~min}$.

\section{Morphometry}

In order to study the distribution of DNA in the total volume of $X Y$ bodies and in the chromosomal axes of the sex chromosomes, DNA immunolocalization was performed in 4 rats. Images of $X Y$ bodies were recorded in video tape at $X 20000$. The numerical density of the gold grains (Number of granules/Area) was estimated in both compartments. The numerical density is an unbiased estimate of the fraction of the volume of the container occupied by the content (Underwood, 1970). All digital procedures were carried out in a PC microcomputer provided with a digitizing and image pro- cessing board (MVP 1024, Matrox) an SVGA monitor and a multisynchronic color monitor connected with the MVP board. The software was Imagenia 5000 from Biocom.

\section{Results}

\section{General morphology}

Compact chromatin is scarce during leptotene and zygotene. The nuclear space is mainly occupied by extended chromatin and ribonucleoprotein (RNP) particles. At late zygotene the chromatin around the forming chromosome axes of $X$ and $Y$ chromosomes begins to fold. Serial sectioning shows that this process may begin in the internal region of the chromosomes (Figure 1). Fibrils of extended chromatin not yet incorporated in the forming body are continuous with filaments located inside the compact regions (Figure 1e). The chromatin of the compacted regions partially keeps the parallel arrangement and the loops present in the chromosomes before compaction (Figure 1e). In the process of incorporation the chromatin is associated with RNP particles as perichromatin granules and fibrils (Monneron and Bernhard, 1969) (Figure If). In early pachytene, when autosomes are completely paired, however, the axes of $X$ and $Y$ chromosomes are aligned parallel but not paired (Figure 2a). A short synaptonemal complex (about $1 \mu \mathrm{m}$ in length) is formed in advanced pachytene stage (Figure $2 b$ ). The non-paired regions of the chromosomal axes diverge from the extremity of the SC and describe curved path inside the XY body.

The chromatin fibrils acquire a fibrogranular or beads-on-a-string structure during maturation of the $X Y$ body. Irregular filaments of chromatin, probably corresponding to loops not contained in the thickness of the section, extend from chromosomal axes and lateral elements of the synaptonemal complex (Figure 2b). The degree of compaction of the chromatin of the $X Y$ body is never as high as that of the clumps of compact chromatin formed by autosomes (Figure 2 a).

The mature $X Y$ body is a circumscribed structure clearly different from the surrounding structures. Occasionally the beaded fibrils are in continuity with external chromatin, which frequently presents loops of different length (Figure $2 \mathrm{c}$ ). Transcriptionunit-like filaments surrounded by pediculate perichromatin granules in the process of formation 


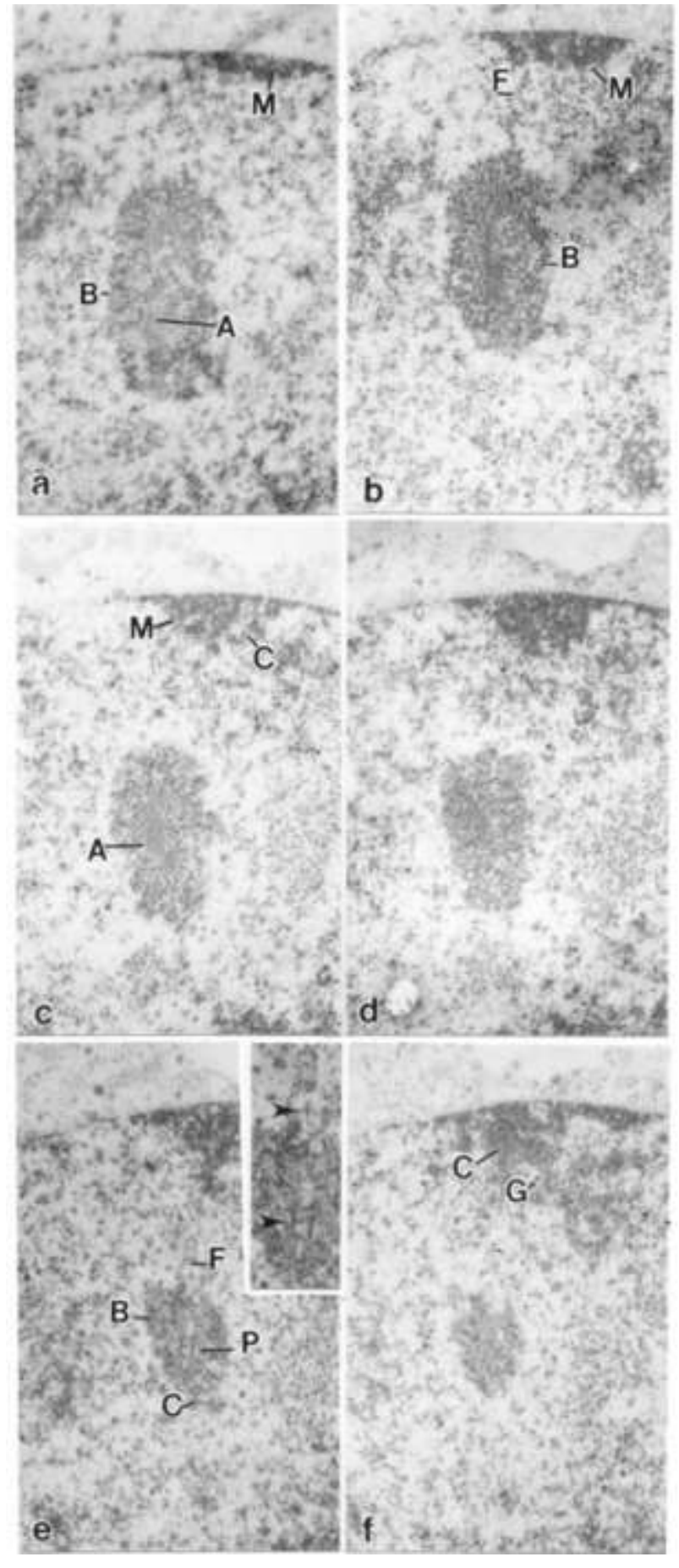

Figure 1. Serial sections of a forming $X Y$ body. General staining. $X 14$ 500. a - A body of semi-compact chromatin (B) is located in the nucleoplasm without apparent continuity with the clumps of chromatin associated with the nuclear membrane (M). A chromosomal axis (A) is visible inside the chromatin body. $b$ - Irregular filaments (F) are present in the gap between the body of semi-compact chromatin (B) and the clump associated with the nuclear membrane (M). C - The chromosomal axis is transversally sectioned (A). Irregular filaments of semi-compact chromatin (C) are located in the nuclear periphery near the clump of compact chromatin (M). d - The axial element is not longer visible. e - The body of semi-compact chromatin (B) is smaller. A pair of parallel fine fibrils (F) extends from the body of semi-compact chromatin. There is a pair of parallel fibrils $(P)$ inside the body of semi-compact chromatin (B). Internal fibrils of the body are in continuity with external filaments (C). The inset shows the fibrils (arrow heads) at a larger magnification X23,000. $f$ - Perichromatin granules (G) are associated with peripheral chromatin (C). can also be seen associated with external extensions of the filaments of the XY body (Figure. $2 \mathrm{~d}$ ).

One or two nucleoli are frequently found adjacent to the $X Y$ body (Figure $2 b$ ). Both structures are separated by a thin space and they are not interwoven (Figure 2b).

\section{Distribution of RNA}

In the mature $X Y$ bodies of spermatocytes in pachytene stage the chromosomal axes and the synaptonemal complex are the only structures contrasted positively by EDTA procedure or by the terbium method (Figure 3, 4, 5). In situ hybridization carried out using a genomic probe without DNA denaturalization of the sample, demonstrates that RNA is localized in the chromosomal axes but not in the filaments of chromatin of the $X Y$ body (Figure 6). In early stages of the meiotic prophase few RNP structures are located at the periphery of the sex body. However, in late pachytene and early diplotene numerous RNP fibrils similar to the perichromatin fibrils described by Monneron and Bernhard (1969) appear surrounding it (Figure 4).

\section{Localization of DNA}

Osmium ammine method shows that the $X Y$ body is mainly formed by a network of positively stained fibrils (Figure 7) corresponding to the beaded fibrils described by general staining methods. As shown by general staining methods, the spatial density of the chromatin in the $X Y$ body is not as high as that of the standard clumps of compact chromatin. The meshes of the network are larger than in the clumps and the distribution of the fibrils shows some features reminiscent of the configuration the chromatin in leptotene and early zygotene. The continuities of the filaments of the XY body with external chromatin fibrils indicate that the semi-compact chromatin of the sex chromosomes may keep an extended configuration outside the sex body (Figures $2 \mathrm{c}$ and 7 ). On the other hand the chromatin of the body does not penetrate into the adjacent nucleoli (Figure 7).

Statistical estimation of the DNA distribution density in the chromosomal axes or the lateral elements of the SC and in the semi compact chromatin of the $X Y$ body, was achieved using immunoelectron microscope localization. Table 1 shows that the gold grains are less concentrated in the axial cores and in the lateral elements of the SC than in the surrounding chromatin. 


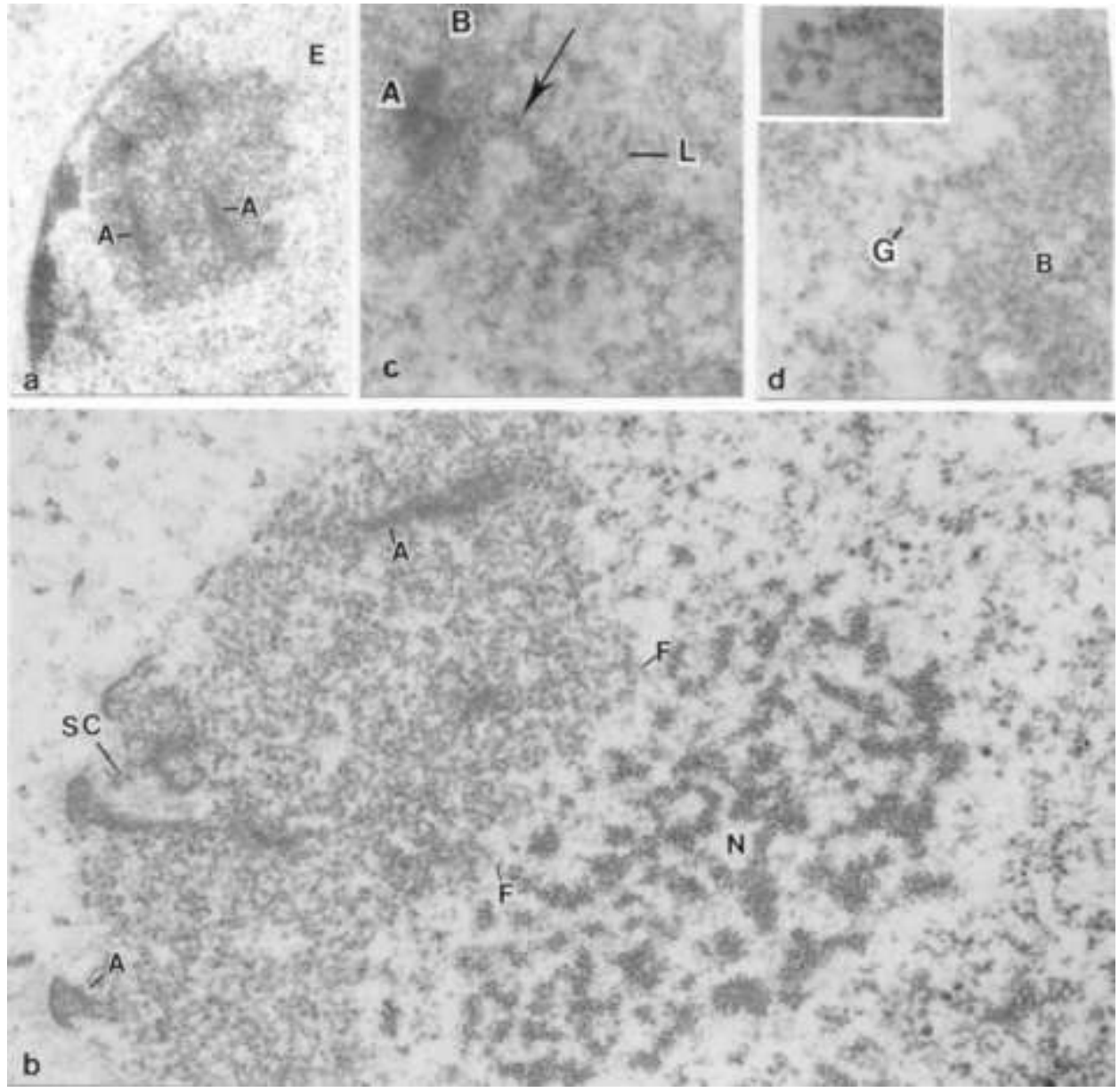

Figure 2 - a. Early pachytene. General staining. The axial elements (A) are not yet paired. The fibrogranular components of the $X Y$ body are continuous with external fine filaments (E). Note the difference in the degree of compaction between the peripheral clumps of compact chromatin and the $X Y$ body. X14,000. b. Pachytene. General staining. The fibrils of chromatin present a fibrogranular structure. A short synaptonemal complex (SC) is located inside the body. Beaded chromatin fibrils originate in the lateral elements of the synaptonemal complex and in the unpaired axial elements $(A)$. The nucleolus $(N)$ is closely associated with the $\mathrm{XY}$ body. Fine fibrils $(F)$ bridge the irregular space between the granular region of the nucleolus and the $X Y$ body. X34,000. c. Pachytene. Osmium ammine staining. The chromatin of the $X Y$ body (B) is continuous with external chromatin (arrow), which may be extended in loops (L) of different length. A - Axial element. X15,000. d. Pediculate perichromatin granules $(G)$ are associated with a fine filament bound to the structure of the $X Y$ body (B). X63,000. The inset depicts the transcriptionunit-like group of pediculate perichromatin granules surrounding an axial fibril. x77,000.

\section{Immunolocalization}

The immunolocalization of DNA corroborates that most of the sex body is formed by fibrils of chromatin (Figure 8). However, chromosomal axes and lateral elements of the synaptonemal complex are labeled to a lesser extent.

The localization of hnRNP shows that the $X Y$ body is practically free of labeling. As expected, the RNP particles located in the nucleoplasm and those surrounding the sex body are labeled (Figure 9a). RNA polymerase II is also absent from the $X Y$ body. The gold grains are located in nearby areas of extended chromatin associated with RNP particles (Figure 9b). The immunolocalization of the trimethyl-guanosin cap, shows that the snURNAs are absent from the $X Y$ body (Figure 9c).

The recombination protein $\mathrm{Dmcl}$ is localized at nodules associated with the axes of sex chromosomes (Figure 3), similar to those present in autosomes. The rest of the sex body is not labeled over the background level (Figure 4).

\section{Discussion}

The formation of the $X Y$ body at the leptotenepachytene transition involves the compaction of the extended chromatin surrounding the forming chromosomal axes. Compaction starts in the zygotene stage when the chromosome axes are formed by fine filaments of chromatin surrounded by loops. This configuration is conserved to some extent and can be discerned in the internal fine structure of the sex body. Compaction begins at one or two regions of the chromosomes. The filaments of chromatin progressively acquire a fibrogranular structure. At the same time these fibrogranular filaments fold forming a three-dimensional meshwork which is never so tight as that of the clumps of compact chromatin found in meiotic prophase or somatic interphase nuclei. These observations as a whole indicate that the process compaction of sex chromosomes is different from the reversible changes extended-compact-extended chromatin found in 
O.M. Echeverria et al.

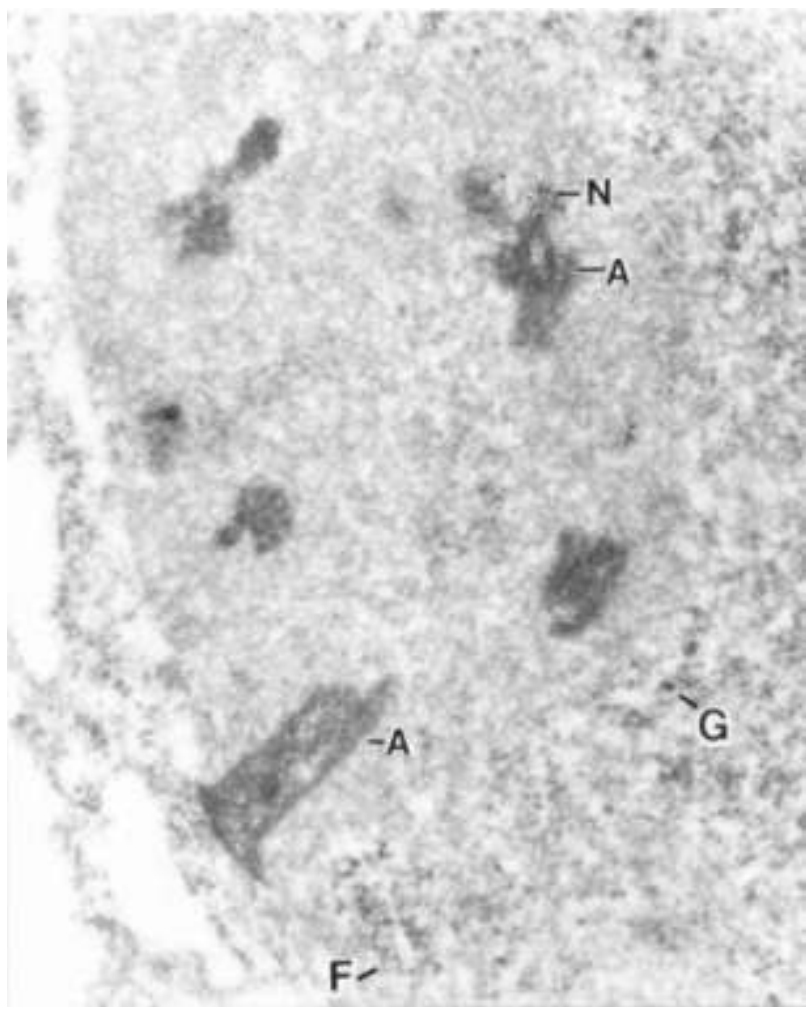

Figure 3. Advanced pachytene. EDTA procedure preferential for RNP. Labeled with anti Dmc1 recombination protein. The only component of $X Y$ body stained is the axial element (A). Numerous positively contrasted fibrils (F) and granules (G) are located at the border of the sex body and also dispersed in the nucleoplasm. A cluster of gold grains labels a nodule associated with an unpaired axial element (N). X44,000.

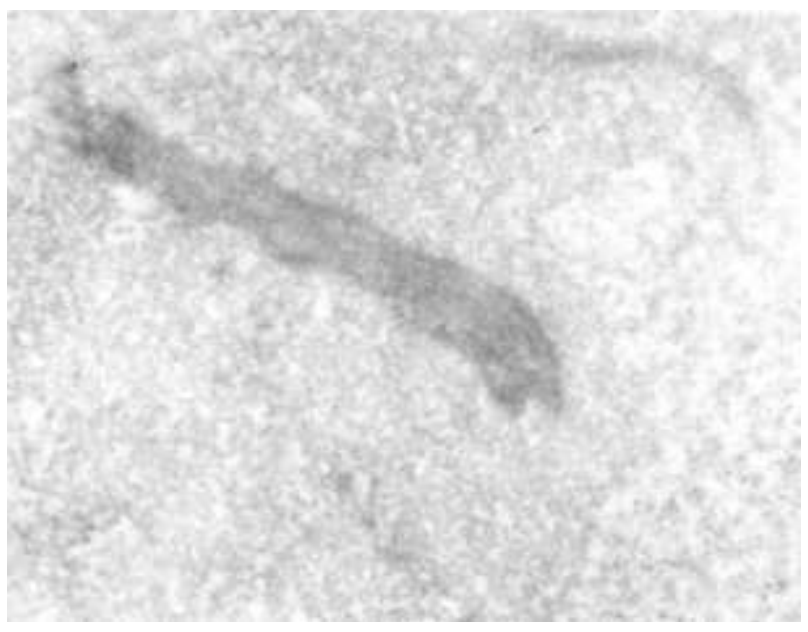

Figure 5. XY body of a spermatocyte in early pachytene. A ribbon-like axial element is stained by terbium method for RNA. The rest of the $X Y$ body is not contrasted. $x 28,000$.

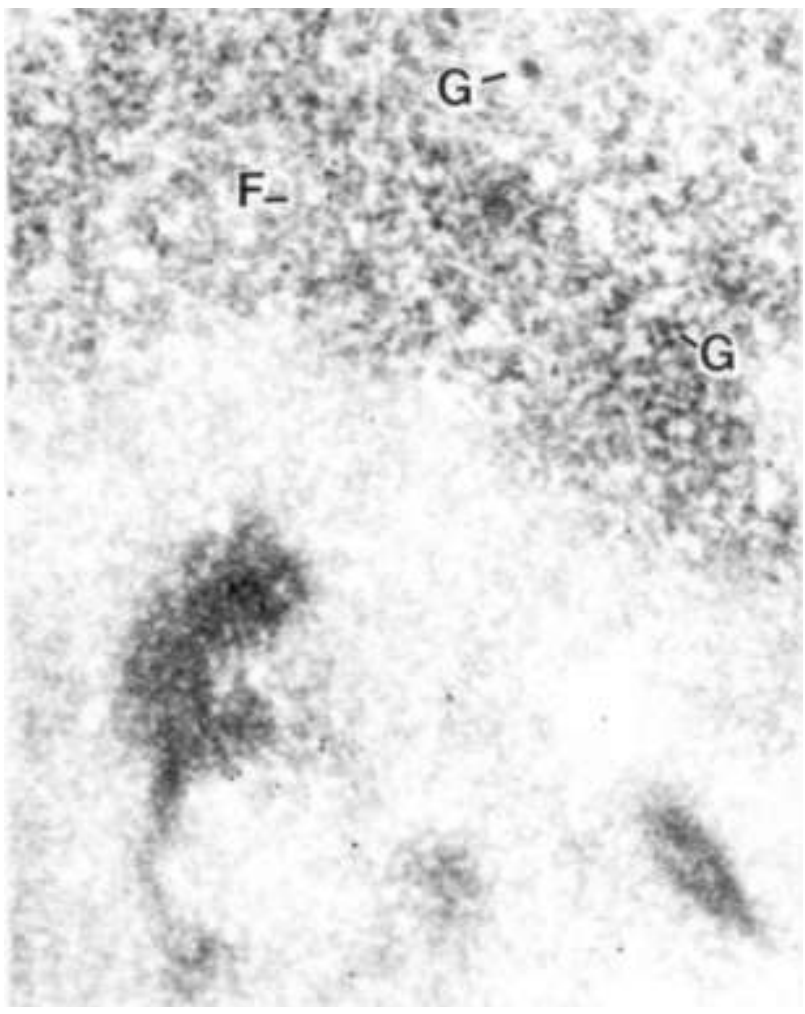

Figure 4. EDTA staining. Labeled with anti recombination protein. In late pachytene and early diplotene positively contrasted fibrils (F) and granules $(G)$ surround the $X Y$ body. The axial elements are dark stained. The chromatin of the sex body is not labeled over background level except at the nodules depicted in the preceding figure. $X 53,000$.

interphase nuclei during drastic changes in the rate of transcription (Vázquez-Nin et al., 1978).

In leptotene and a part of the zygotene stage the chromatin of sex chromosomes, as that of autosomes, is associated with RNP fibrils and granules. During the compaction of the chromatin the RNP structures disappear from the maturing $X Y$ body. The compaction of chromatin and the disappearance of RNA polymerase II and different types of

Table 1.

\begin{tabular}{lccc}
\hline \hline & $\begin{array}{c}\text { Mean numerical } \\
\text { density }\end{array}$ & $N$ & $\begin{array}{c}\text { Standard } \\
\text { deviation }\end{array}$ \\
\hline Chromatin & 70.6 & 13 & 19.4 \\
Chromosomal axes & 8.2 & 13 & 5.8 \\
\hline
\end{tabular}

$t=11.4 ;$ Significance level $=3.7 \times 10^{11}$ 


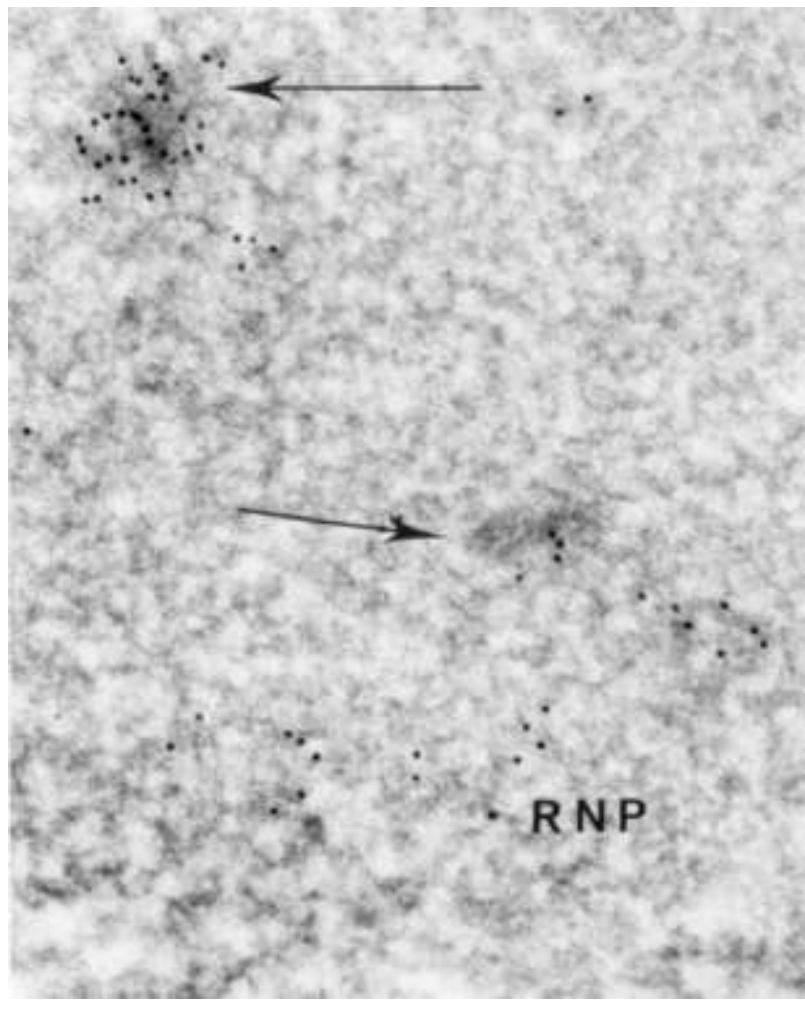

Figure 6. In situ hybridization performed without denaturation of the DNA of the sample in order to localize exclusively RNA. General staining. Most of gold granules are associated with axial elements (arrows). The rest of the body is not labeled over background level. The RNP particles surrounding the XY body (RNP) are labeled. $x 78,000$.

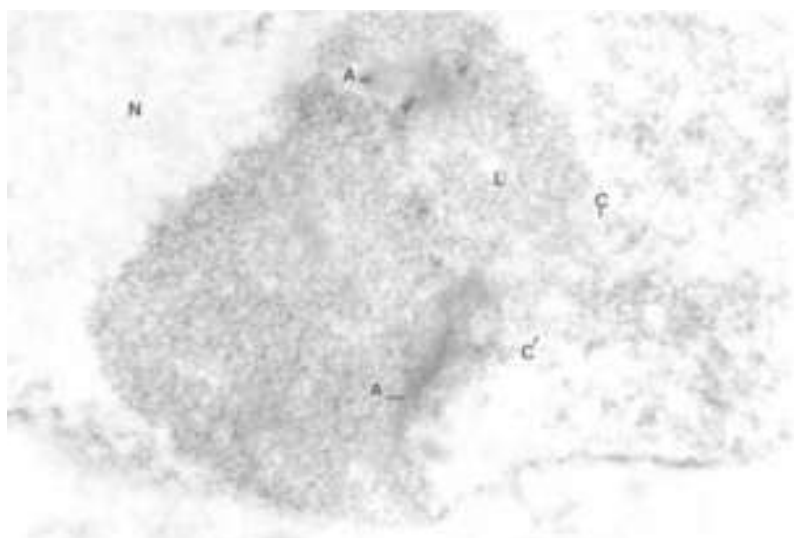

Figure 7. Osmium ammine method for DNA. The main structural component of the XY body is a mesh of chromatin filaments. These irregular fibrils are folded forming loops of different length (L). The homogeneous gray areas correspond to the axial elements of the chromosomes (A). Some internal filaments of the body are continuous with external extended chromatin (C). The chromatin of the sex chromosomes does not penetrate inside the nucleolus $(\mathrm{N}) . \times 46,000$.

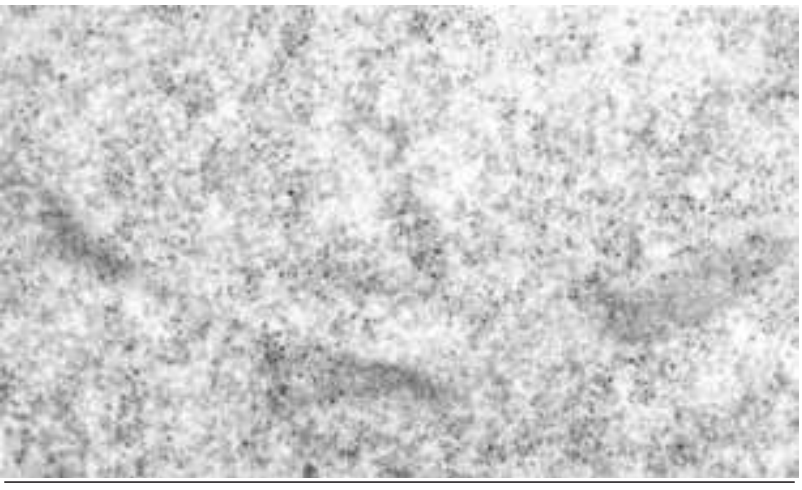

Figure 8. Pachytene. XY body labeled with anti-DNA mAb. The gold grains are densely distributed over the entire section of the body. The dark stained sections of the axial elements are labeled to a lesser extent. x124,000.

RNP particles occur during formation of the $X Y$ body suggesting that both phenomena may be related, as was postulated by Solari (1974). However, the precise timing of these phenomena remains to be studied. Important changes in the protein composition of the sex chromosomes take place during the formation of the body (Kralewski and Benavente, 1997). Some of these proteins may be involved in producing heterochromatin-like condensation of the chromatin and also transcription inactivation (Bauer et al., 1998).

During most of the pachytene the autosomes present a region of dense chromatin located in the vicinity of the SC, which is associated with few RNP structures, and a peripheral region of extended chromatin supporting intense transcription. In this stage, EDTA and terbium methods, as well as in situ hybridization, show that the chromatin of the $X Y$ body is not associated with any RNP structure. Immunolocalizations demonstrate that the mature body lacks RNA polymerase II, hnRNPs, and small nuclear uridine rich RNA involved in pre messenger splicing. This allocyclic behavior has been previously analyzed (for reviews see McKee and Handel, 1993; Solari, 1994). However, our detailed ultrastructural observations provide evidence indicating that not all the chromatin of the sex chromosomes is transcriptionally inactive. Osmium-ammine methods for DNA and general staining procedures demonstrate the presence of continuities of the internal fibrils with external extended filaments of chromatin at the periphery of the sex body. That is, extensions of the filaments of chromatin of the $X Y$ body to the surrounding nucleoplasm. These obser- 
vations indicate that the chromatin of the sex chromosomes is not completely contained inside the $X Y$ body. These external fibrils are frequently associated with perichromatin fibrils and perichromatin granules in course of formation. The immature perichromatin granules are recognized by the presence of a stalk or pedicle linking the granule with the chromatin, as previously described (Vázquez$\mathrm{Nin}$ et al., 1996). Perichromatin fibrils are composed by newly transcribed pre-mRNA, frequently in process of splicing (Fakan and Bernhard, 1973; Fakan et al., 1976; Fakan et al., 1984; Fakan et al., 1986; Cmarko et al., 1999). The association of chromatin filaments of the sex chromosomes with these forming RNP structures strongly suggests that part of the DNA of these chromosomes is transcribed in meiotic prophase. However, the transcribed sequences are located outside the sex body. As RNA polymerase II is absent from the XY body, it represents a site of general repression of transcription of pre-mRNA. Thus, the expression of important genes of $X$ chromosome may only take place outside the sex body.

The earliest immunocytochemical determination of the presence of the recombination proteins Rad51/Dmcl during yeast gametogenesis coincides with the biochemical detection of double strand breaks (Bishop, 1994). Most authors agree that in mammalian meiosis Rad51/Dmcl is present in nodules associated with axial elements during pairing, and that after synapsis their number decreases sharply (Moens et al., 1997; Barlow et al., 1997; Plug et al. 1998). Furthermore, Rad51/Dmcl was found to colocalize with the protein components of the chromosomal axes and of the lateral elements of the SC (Moens et al., 1997; Barlow et al., 1997; Plug et al. 1998). The finding of Rad51/Dmcl in nodules associated with not pairing and not recombining chromosomal regions of the sex chromosomes may be interpreted in two ways: 1 ) The presence of this protein in the $X Y$ body is not associated with any of these processes; 2 ) The protein is still there because there is no homologous region and pairing cannot take place.

Figure 9 [right column]. a. $X Y$ body of a spermatocyte in advanced pachytene labeled with anti-hnRNP. The label is associated with structures external to the body (F). The XY body is not labeled. X 27000. b. Advanced pachytene. Immunolocalization of RNA polymerase II. The gold grains label pachytene. Immunolocalization of RNA polymerase II. The gold grains label of the body (C) is not labeled. X47,000. c. Immunogold localization of the trimethyl-guanosin cap of the snURNA. Most of the gold grains are associated with nucleoplasmic RNP fibrils. The chromatin filaments of the sex body $(\mathrm{C})$ are not labeled. $\mathbf{X} 50,000$.
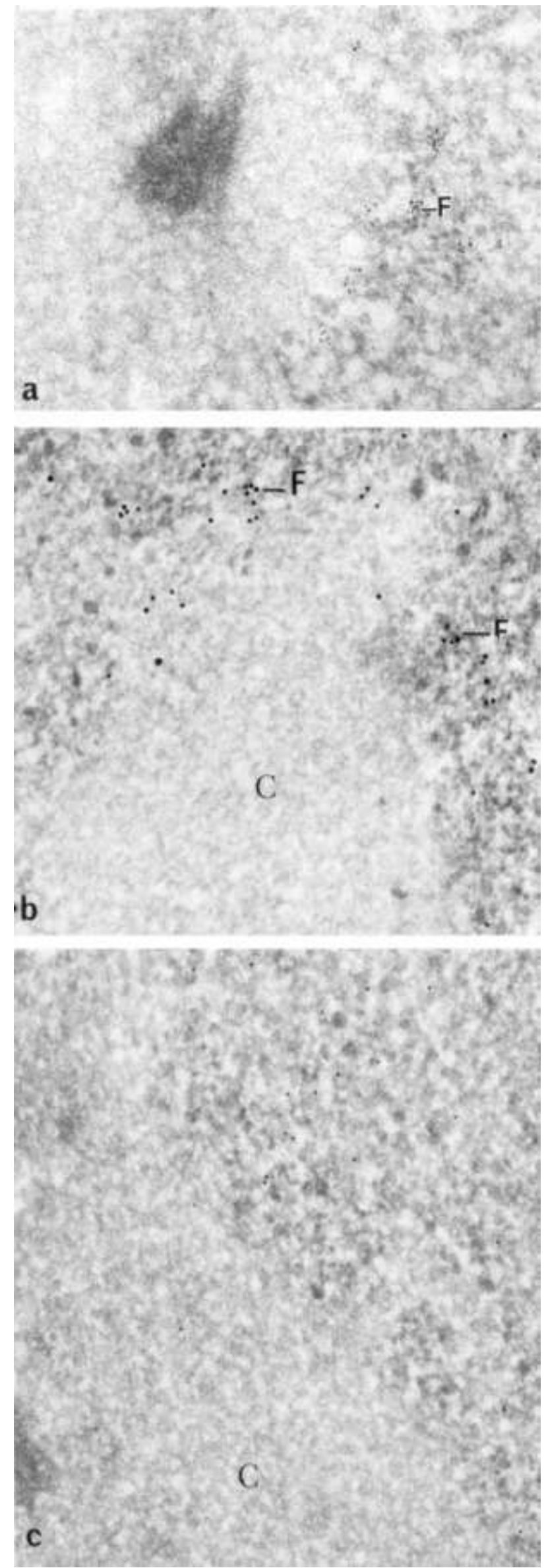
Nucleoli are frequently adjacent to the $X Y$ body in rat and guinea pig spermatocytes. However, both structures are separated by a thin clear space occasionally bridged by thin fibrils, faintly contrasted by general staining methods. EDTA and terbium procedures for RNA show that the XY body and the nucleoli do not share RNP structures. The osmiumammine procedure demonstrates that the chromatin of the sex body does not penetrate in the nucleolus. The fine fibrils bridging the space between both structures are probably fine protein filaments that are not contrasted by staining methods for nucleic acids. Filament resistant to long RNase treatments and depicting a low signal of phosphorus or not at all (deficient in nucleic acids), were previously described in the nucleoplasm of the salivary gland cells of Chironomus thummi (Vázquez-Nin et al., 1993, 1997) and rat hepatocytes (Vázquez-Nin et al., 1996). It is tempting to speculate that these filaments are components of the nuclear matrix that can be seen in situ in electron energy spectroscopic images and occasionally in standard preparations. These results indicate that the spatial relation between the $X Y$ body and the nucleolus is only juxtaposition, just proximity, defined as a secondary association by Solari (1974). However, the proximity of one or two nucleoli to the sex body in rat and guinea pig spermatocytes is very frequent. This type of association is present in mammals in which the nucleolus organizer is not located in the sex chromosomes (Solari, 1974).

It is interesting to note that the only structures of the pachytene $X Y$ body that exhibit a positive contrast with the EDTA and terbium methods for RNP are the chromosomal axes and the lateral elements of the SC. These results confirm previous observations indicating the presence of RNA in the corresponding elements of the autosomes (Vázquez-Nin and Echeverría, 1976). RNA persists in the axial elements of sex chromosomes much longer than in autosomes. In the latter, the RNA is no longer detected in mid-pachytene, while in the $X Y$ body it is detected in unpaired axial structures until final pachytene. These observations suggest that the presence of RNA in the chromosomal axes may be related with the formation of the SC. Once the complex is mature the RNA disappears from the lateral elements, while in unpaired regions it persists much longer.

\section{Acknowledgements}

The authors like to thank Ernestina Ubaldo for their excellent technical assistance, Alberto Carlos for photographic work and to M.V.Z. Mario Soriano for animal care.

This work was supported by grant I/74 348 of the Volkswagen-Stiftung, UNAM PAPIIT IN227398, CONACYT 36450-N grants, and a Purina grant for animal food.

\section{References}

Armstrong SJ, Hultén MJ, Keohane AM, Turner BM.Different strategies for $X$-inactivation in germinal and somatic cells: histone 4 underacetylation does not mark inactive $\mathrm{X}$-chromosome in mouse male germline. Exp Cell Res 1997;230:399-402.

Barlow AL, Benson FE, West SC, Hultén MA. Distribution of the Rad51 recombinase in human and mouse spermatocytes. EMBO J 1997;16:5207-15.

Baumann P, Benson FE, West SC. Human Rad51 protein promotes ATP-dependent homologous pairing and strand transfer reactions in vitro. Cell 1996;87:737-66.

Bauer VM, Schneider-Hirsch S, Reinherdt S, Benavente R, Maelicke A. The murine nuclear orphan receptor GCNF is expressed in the $X Y$ body of primary spermatocytes. FEBS Lett 1998;208:208-14.

Benson FE, Stasiak A, West SC. Purification and characterization of the human Rad51 protein, an analogue of E. coli ReA. EMBO J 1994;13:5764-71.

Bernhard W. A new staining procedure for electron microscopical cytology. J Ultrastruct Res 1969;27:250-65.

Biggiogera M, Fakan S. Fine structural specific visualization of RNA on ultrathin sections. J Histochem Cytochem 1998;46:389-95.

Biggiogera M, Fakan S, Kaufmann SH, Black A, Shaper JH, Bush H. Simultaneous immunoelectron microscopic visualization of protein B23 and C23 distribution in the HeLa cell nucleolus. J Histochem Cytochem 1989;9:1371-4.

Bishop DK. RecA homologs DMC1 and RAD51 interact to form multiple nuclear complexes prior to meiotic chromosome synapsis. Cell 1994;79:1081-92.

Clermont Y. Cycle of the seminiferous epithelium of the guinea pig. Fert Steril 1960;6:563-73.

Cmarko D, Verschure PJ, Martin TE, Dahmus ME, Krause S, Fu XD, et al. Ultrastructural analysis of transcription and splicing in the cell nucleus after bromo-UTP microinjection. Mol Biol Cell 1999;10: 211-23.

Cogliati R, Gautier A. Demonstration of DNA and polysaccharides using a new "Schiff type" reagent. C R Acad Sci Hebd Seances Acad Sci D 1973;276:3041-4.

Cook PR. The transcriptional basis of chromosome pairing. J Cell Sci 1997;110:1033-40.

Fakan S, Bernhard W. Nuclear labeling after prolonged $3 \mathrm{H}$-uridine incorporation as visualized by high resolution autoradiography. Exp Cell Res 1973;79:431-44.

Fakan S, Puvion E, Spohr G. Localization and characterization of newly synthesized nuclear RNA in isolated rat hepatocytes. Exp Cell Res 1976;99:155-64.

Fakan S, Leser G, Martin TE. Ultrastructural distribution of nuclear ribonucleoproteins as visualized by immunocytochemistry on thin sections. J Cell Biol 1984;98:358-63.

Fakan S, Leser G, Martin TE. Immunoelectron microscope visualization of nuclear ribonucleoprotein antigens within spread transcription complexes. J Cell Biol 1986;103:1153-7.

Jones RE, Okamura CS, Martin TE. Immunofluorescent localization of the proteins of nuclear ribonucleoprotein complexes. J Cell Biol 1980;86:235-43.

Kierszenbaum AL, Tres LL. Nucleolar and perichromosomal RNA synthesis during meiotic prophase in the mouse testis. J Cell Biol 1974; $60: 39-53$. 
Kim WY, Dahmus ME. Immunocytochemical analysis of mammalian RNA polymerase II subspecies. Stability and relative in vivo concentration. J Biol Chem 1986;261:14219-25.

Kleckner N. Meiosis: how could it work? Proc Natl Acad Sci USA 1996;93:8167-74.

Kleckner N, Padmore R, Bishop DK. Meiotic chromosome metabolism: one view. Cold Spring Harbor Symposia on Quantitative Biology 1991;56:729-43.

Kleckner N, Weiner BM. Potential advantages of unstable interactions for pairing of chromosomes in meiotic, somatic, and premeiotic cells. Cold Spring Harbour Symposia on Quantitative Biology 1993; 63:553-5.

Kralewski M, Benavente R. XY body formation during rat spermatogenesis: an immunocytochemical study using antibodies against $X Y$ body-associated proteins. Chromosoma 1997;106:304-7.

Kralewski M, Novello A, Benavente R. A novel Mr 77,000 protein of the $X Y$ body of mammalian spermatocytes: its localization in normal animals and Searle's translocation carriers. Chromosoma 1997; 106:160-7.

Leblond $C P$, Clermont Y. Definition of the stages of the cycle of the seminiferous epithelium in the rat. Ann N Y Acad Sci 1952; 55:54869.

Loidl J. The initiation of meiotic chromome pairing: the cytological view. Genome 1990;33759-78.

López-Velázquez L, Segura-Valdez L, Alcántara-Ortigoza MA, Jiménez-García LF. Localization of intranuclear RNA by electron microscopy in situ hybridization using a genomic probe. Arch Med Res 1998;29:185-90.

Marahrens Y, Panning B, Dausman J, Strauss W, Jaenisch R. Xist-deficient mice are defective in dosage compensation but not spermatogenesis. Genes Dev 11 1997;156-66.

McKee BD. The license to pair: identification of meiotic pairing sites in Drosophila. Chromosoma 1996;105:135-41.

McKee BD, Handel MA. Sex chromosomes, recombination, and chromatin conformation. Chromosoma 1993;102:71-80.

Moens PB. Molecular perspectives of chromosome pairing at meiosis. BioEssays 1994;16:101-6.

Moens PB, Chen DJ, Shen Z, Kolas N, Tarsounas M, Heng H HQ. Spyropoulos B. Rad51 immunocytology in rat and mouse spermatocytes and oocytes. Chromosoma 1997; 106:207-15.

Monneron A, Bernhard W. Fine structural organization of the interphase nucleus of some mammalian cells. J Ultrastruct Res 1969; 27:266-88.

Plug AW, Peters AH, Keegan KS, Hoekstra MF, de Boer P, Ashley T. Changes in protein composition of meiotic nodules during mammalian meiosis. J Cell Sci 1998;111:413-23.

Smith A, Benavente R. Meiosis-specific protein selectively associated with sex chromosomes of rat pachytene spermatocytes. Proc Natl Acad Sci USA 1992;89:6938-42.

Smith A, Benavente R. An Mr 51,000 protein of mammalian spermatogenic cells that is common to the whole $X Y$ body and centromeric heterochromatin of autosomes. Chromosoma 1995; 103: 591-6.

Smithies 0, Powers P. Gene conversions and their relation to homologous chromosome pairing. Philos Trans R Soc Lond B Biol Sci 1986;312:291-302.
Solari AJ. The behaviour of the XY pair in mammals. Int Rev Cytol 1974;38:273-317.

Solari AJ. Sex chromosome pairing and fertility in the heterogametic sex of mammals and birds. In: Fertility and chromosome pairing: recent studies in plants and animals (Ed. Gillies C.B.). CRC Boca Raton, USA: 1969; p. 77-107.

Solari AJ. Sex chromosomes and sex determination in vertebrates. CRC Press, Boca Raton, USA: 1994.

Solari AJ, Tres LL. The three-dimensional reconstruction of the $X Y$ chromosomal pair in human spermatocytes. J Cell Biol 1970; 45:43-53.

Sybenga J. What makes homologous chromosomes find each other in meiosis? A review and an hypothesis. Chromosoma 1999;108:20919.

Turner JM, Mahadevaiah SK, Benavente R, Offenberg HH, Heytig C, Burgoyne PS. Analysis of male meiotic "sex body" proteins during $X Y$ female meiosis provides insights into their functions. Chromosoma 2000;109:426-32.

Underwood E. Quantitative stereology. London, Addison-Wesley, 1970.

Vázquez-Nin GH, Abolhassani-Dadras S, Echeverría OM, Boutinard Rouelle-Rossier V, Fakan S. Phosphorus distribution in perichromatin granules and surrounding nucleoplasm as visualized by electron spectroscopic imaging. Biol Cell 1996;87:171-7.

Vázquez Nin GH, Abolhassani-Dadras S, Echeverría OM, BoutinardRouelle-Rossier V, von Shack ML, Fakan S. Electron spectroscopic imaging analyses of the distribution of phosphorus in Balbiani ring granules and in the surrounding nucleoplasm. Chromosoma 1997; 105:360-8.

Vázquez-Nin GH, Biggiogera M, Echeverría OM. Activation of osmium ammine by S02-generating chemicals for electron microscopy Feulgen-type staining of DNA. Eur J Histochem1995;39:101-6.

Vázquez-Nin GH, Echeverría OM. Ultrastructural study on meiotic prophase nucleus of rat oocytes. Acta Anat 1976;96:218-31.

Vázquez Nin GH, Echeverría 0M, Boutinard Rouelle-Rossier V, Fakan $\mathrm{S}$. A new type of ribonucleoprotein constituent of the polytene nucleus of the salivary gland of Chironous thummi and Ch. tentans. Chromosoma 1993;102:693-9.

Vázquez-Nin GH, Echeverría OM, Fakan S, Leser G, Martin TE. Immunoelectron microscope localization of snRNPs in the polytene nucleus of salivary glands of Chironomus thummi. Chromosoma 1990;99:44-51.

Vázquez-Nin GH, Echeverría OM, Molina E, Fragoso J. Effects of ovariectomy and estradiol injection on nuclear structures of endometrial epithelial cells. Acta Anatomica (Basilea) 1978; 102:308-18.

Weiner BM, Kleckner N. Chromosome pairing via multiple interstitial interactions before and during meiosis in yeast. Cell 1994;77:97791.

Wettstein R, Graver A. Film-supporting frames for mounting sections grids. J Ultrastruct Res 1973;43:436-47. 